\title{
Tabletop Experiment to Verify Magnetic Monopoles
}

\author{
Rainer W. Kühne \\ Recently, we suggested a model of magnetic monopoles hep-ph/9708394). Here we will propose \\ a tabletop experiment to test this model. The verification of the predicted effect would have far- \\ reaching consequences. \\ PACS number: $14.80 . \mathrm{Hv}$
}

Recently, we suggested a quantum field theoretical model of the electromagnetic interaction which includes magnetic monopoles [1]. This model is based on Dirac's original proposal of a two-potential theory [2] that includes the wellknown photon and also Salam's "magnetic photon" [3]. According to this model the cross-section of the magnetic photon depends on the absolute motion of the electric charge with which it interacts.

Such an absolute rest frame is supported by several observations.

(1) General relativity gives rise to an expanding universe and therefore to a finite-sized light zone. The center-ofmass frame of this Hubble sphere can be regarded as a preferred frame.

(2) A preferred motion is given at each point of space by cosmological observations, namely the redshift-distance relation generated by the superposition of the Hubble and the Doppler effect which is isotropic only for a unique rest frame.

(3) A preferred motion is given also by the dipole anisotropy of the cosmic microwave background radiation due to the Doppler effect by the Earth's motion of $370 \mathrm{~km} / \mathrm{s}$ 四.

According to the proposed model this absolute rest frame gives rise to local physical effects. The interaction crosssection of a magnetic photon (with conventional matter in the terrestrial rest frame) is predicted to be smaller than the one of a photon of the same energy, the suppression factor is $(370 / 299,792)^{2} \simeq 10^{-6}$. This means that each reaction that produces photons does also create magnetic photons. Furthermore, magnetic photons are one million times harder to create, to shield, and to absorb (detect) than photons of the same energy.

This model can be tested by a relatively simple experiment. Shoot a laser beam on a metal foil with a thickness of several micrometers and place a photographic plate (or a charge coupled device or a photomultiplier tube) behind the foil to measure the intensity of the penetrating fraction of the beam. Our model predicts the detected intensity to be of the order of $10^{-12}$ times the one of the original laser beam (only a fraction of the magnetic photon part of the beam will be detected, the photon part is entirely absorbed by the metal foil). In contrast to this, the standard model predicts zero intensity. Background effects by scattering can presumably be reduced to several orders below the predicted effect.

It will be of great benefit to perform the proposed experiment. The verification of the predicted effect would have far-reaching consequences.

(1) Evidence for a new gauge boson and a new kind of radiation ("magnetic photon rays").

(2) Indirect evidence for magnetic monopoles.

(3) Evidence for an absolute rest frame that gives rise to local physical effects.

[1] R. W. Kühne, hep-ph/9708394.

[2] P. A. M. Dirac, Proc. Roy. Soc. A 133, 60 (1931).

[3] A. Salam, Phys. Lett. 22, 683 (1966).

[4] G. F. Smoot, M. V. Gorenstein, and R. A. Muller, Phys. Rev. Lett. 39, 898 (1977). 